\title{
EFFICIENCY OF HUMIC ACID ON WHEAT PRODUCTIVITY AND SOME SOIL PROPERTIES UNDER NO- TILLAGE SYSTEM AND WATER IRRIGATION SHORTAGE
}

\section{Gihan . A. Mohamed" and A.S. El- Hamed**}

Soil, Water and Environment Res. Inst . ARC, Giza, Egypt.*

Field Crops ,Res . Inst . ARC , Giza , Egypt

\section{ABSTRACT}

Two field experiments were conducted in the Experimental Farm of Sids Agricultural Research Station, ARC, Beni-Suef Governorate, Egypt on wheat to determine the possibility of reducing tillage processes and irrigation number by added humic acid and its effect on wheat productivity and soil properties after wheat harvest. The experimental design were split -split design, where tillage systems( no tillage, $\mathrm{N} \mathrm{T}$ and conventional tillage , $\mathrm{C} \mathrm{T}$ ) were arranged in main plot, while irrigation numbers $\left(4, \mathrm{I}_{1}\right.$ and $5, \mathrm{I}_{2}$ irrigations $)$ were conducted in sub plot . Whereas ,sub sub plots were occupied by humic acid treatments $\left(0,0 \mathrm{H}_{1}, 12 \mathrm{H}_{2}\right.$ and $24, \mathrm{H}_{3} \mathrm{~kg}$ humic acid ha ${ }^{-1}$ as soil application ). The results indicate that direct sowing (NT) improved soil organic matter content ,bulk density and soil water relations (increased soil available water and decreased wilting point ), while conventional tillage (CT) increased yield and its components of wheat than no tillage .Irrigated wheat plants with five irrigation increased yield and it components than irrigated four irrigation .Increasing humic acid levels were significantly improved soil $\mathrm{pH}$, soil organic matter, soil bulk density, water relations, soil available N,P and $\mathrm{K}$ and yield and its components of wheat. The results of the interactions show that no tillage system with $24 \mathrm{~kg} \mathrm{ha}{ }^{-1}$ humic acid as soil application under irrigated wheat plant four times gave wheat productivity equal to those under conventional tillage and irrigated five times . this means that it could reduced the tillage cost and save one irrigation and improved soil properties and fertility .

Key words: Tillage, irrigation, humic acid ,some soil properties and fertility and

\section{INTRODUCTION} wheat productivity.

Total wheat consumption has increased drastically over the past many decates,

partially due to the an overall population growth . Accordingly, wheat production do not meet the local consumption, also efforts had been pared for increasing yield per unit area using improved varieties and good agronomic practices .

Tillage is the most important operation for crop production. Strudley et al (2008) mentioned that ,tillage is defined as mechanical operations of soil for crop growing ,which affects many physical soil properties such as soil temperature as well as soil water retentions and infiltration rate .Irshad et al., (2007) reported that there are two types of tillage, conventional tillage and conservation or no tillage . Conventional tillage includes many operation, which aims to integrate plant residues and organic manures into soil (Vakali et al 2011), while conservation or no tillage is

Fayoum J. Agric. Res. \& Dev., Vol. 34, No.2 July, 2020 
EFFICIENCY OF HUMIC ACID ON WHEAT PRODUCTIVITY 67

the method of seedbed preparation that contains plant residues mulch and left the surface of soil roughness as the basic feature (Lal,1990). Many number of serial passes are required for land preparation to left soil surface bare and uncovered till crop grow and development, consequently exposed the land for wind and precipitation resulting nutrient loss and soil erosion (Wells et al., 2013) . In addition, Hobbs et al (2008) showed that conventional tillage is affecting soil productivity due to erosion and organic matter degradation. The conventional tillage is high in cost , complex ,slow, labor intensive and requires more energy, therefore it is not ecofriend system (Tebrugge and during , 1999). They added that the ecological disadvantage of this system include :Soil compaction caused by recurrent machinery use over the soil ,reduction in soil organic matter as well as soil erosion .On the other hand, reduced or no tillage is ecofriendly system which requires less passes of the land, saved time, reduced cost ,energy, soil erosion , nutrient loss through leaching . In this concern, Mosaddeghi et al.,(2017) indicated that no tillage enhanced physical properties comparing with conventional tillage

Irrigation is one of the most important factors, which has played the greatest role in crop production .It supply soil moisture at different growth stages to obtain economically and maximum yield of crops. Baquedano and Castillo (2006) reported that water is most important in various metabolic activities, where its deficiency caused water stress in vegetation in combination with plant, climate and soil . Plant can tolerant water stress by maximizing water absorption through absorbing ground water by deep roots or minimizing water loss , etc (Kozlowski and Pallardy ,2002) .Moreover , El- Shanhorey (2015) mentioned tha water is the major component in plant tissue, where it consider the reagent in leaf chemical reaction, a solvent for mineral and metabolites and its translocation for all enlargement and division He added that many of physiological activities needed for growth are negatively influenced under severe water stress, resulting in death of plant .

Humic acid is a major constituent of humic materials, which resulted from biological decomposition of organic matter. It contain carboxyl and phenolic groups , that it can functionally as di or tri basic acid (Zhang et al ,2013 ). These organic complex affect soil physical and chemical properties (Schanitzer , 1992 ), enhanced nutrient uptake (Mackowiak et al ,2001), increased root length (Cenellas et al ,2002) and improved plant fresh and dry weight (Chen et al ,2004). In addition, Salman et al (2005) indicated that humic acid supply plants with nutrients, improved soil fertility, help the plant to tolerant the drought enhancing the water relations of soil , improved seed germination, improved soil drainage and aeriation, increased protein and nutrient content in most plants and enhanced microorganisms activity ; consequently increased plant productivity .

This investigation was conducted to study the effect of tillage system and humic acid application under water stress conditions on physical and chemical soil properties after wheat harvest as well as wheat productivity .

Fayoum J. Agric. Res. \& Dev., Vol. 34, No.2 July, 2020 
Gihan . A. Mohamed et al.,

\section{MATERIALS AND METHODS}

Two field experiments were conducted at Sids Agric. Res. Station, Beni-Suef Governorate, ( Lat $.29^{\circ} 04 \mathrm{~N}$, Long $.31^{\circ} .06 \mathrm{E}$ and $30.40 \mathrm{~m}$ above the sea level ) ARC, Egypt during the two successive seasons of 2017/2018 and 2018/2019 to study the effect of irrigation numbers and humic acid application under two tillage system on wheat productivity and soil properties and fertility after harvest. Tillage treatments $(T)$ were no tillage $\left(T_{1}\right)$ as direct sowing and one tillage $\left(T_{2}\right)$ as one pass of a disk, while irrigation treatments (I) without put planting irrigation in consideration were :four irrigations $\left(\mathrm{I}_{1}\right)$ at 20,40,70 at 100 days ; and five irrigation $\left(\mathrm{I}_{2}\right)$ at 20,40,70,100, and 125 days from planting. Whereas, humic treatments $(\mathrm{H})$ were : $0.0\left(\mathrm{H}_{1}\right), 10.0\left(\mathrm{H}_{2}\right)$ and $24\left(\mathrm{H}_{3}\right) \mathrm{kg} \mathrm{h}^{-1}$ humic acid . The experimental soils were clay in texture for surface soil sample having $\mathrm{pH}$ of 8.01 and 8.0 ,EC of 1.23 and $1.35 \mathrm{dSm}^{-1}$ and soil organic matter of 2.13 and $2.36 \%$, as well as bulk density of 1.22 and $1.20\left(\mathrm{gm}^{-3}\right)$, soil available water of $22.57 \mathrm{and} 22.26\left(\mathrm{ug} \mathrm{g}^{-1}\right)$, witting point of 20.40 and 20.11 (ug g-1 ) and soil available N,P and $\mathrm{K}$ of 23.5 and $21.1 ; 15.2$ and 16.7 ; and 185 and $192 \mathrm{ug} \mathrm{g}^{-1}$, in both growing seasons, respectively (according to A.O.A.C , 1980 ) . The experimental design were split -split in completely randomized block in four replications. The tillage treatment were arranged in the main plots, while treatments were applied in sub - sub plots. Wheat grains (c.v.Beni - Suef 5 ) were sowing at 15 and 20 November in both seasons, respectively in plots $\left(10.5 \mathrm{~m}^{2}=1 / 450\right.$ ha $)$. Nitrogen and potassium fertilizers were added at rate of 179 $\mathrm{kg} \mathrm{N} \mathrm{ha}^{-1}$ and $57 \mathrm{~kg} \mathrm{~K} \mathrm{ha}^{-1}$ as ammonium nitrate $(33.5 \% \mathrm{~N})$ and potassium sulphate $48 \mathrm{~kg} \mathrm{~K} \mathrm{ha}^{-1}$ in two equal doses, the first before the first irrigation and the second before the second one. Also, phosphorus fertilizer was added before sowing during the land preparation at rate of $53 \mathrm{~kg} \mathrm{p}_{2} \mathrm{O}_{5} \mathrm{ha}^{-1}$ as superphosphate fertilizer $\left(15.5 \% \mathrm{P}_{2} \mathrm{O}_{5}\right)$. Other cultural practices for wheat production were done as in district . At 75 days from sowing ten plants were taken to determine some wheat growth parameters such as plant height, dry weight plant ${ }^{-1}$ and number of tillers plant ${ }^{-1}$; also ten plants were randomly taken to measure some yield components, namely, number of spikes $\mathrm{m}^{-2}$, number of grains spike ${ }^{-1}$ and 1000 - grain weight $(\mathrm{g})$. Also , grain and straw yield were measured for all plot and converted to ton ha ${ }^{-1}$. Grain and straw samples were taken to determine N,P and K contents ( as the method described by Chapman and Pratt , 1961). Surface soil samples were taken from each plot before sawing and after wheat harvest to determine some soil properties, i.e., $\mathrm{pH}$, EC , soil organic matter, soil available $\mathrm{N}, \mathrm{P}$ and $\mathrm{K}$ as well as soil bulk density, available water and wilting point (according to A.O.A.C.,1980). All collected data were subjected to the statistical analysis according the procedure described by Gomez and Gomez (1993). Means of treatments were compare using Duncan Multiple Range Test at 5\% level of probability .

Fayoum J. Agric. Res. \& Dev., Vol. 34, No.2 July, 2020 
EFFICIENCY OF HUMIC ACID ON WHEAT PRODUCTIVITY 69

\section{RESULTS}

\section{Physical soil properties:}

Data in Table (1) represent the effect of tillage system , irrigation number and humic acid application on soil properties ,i.e., $\mathrm{pH}$; EC , soil organic matter , bulk density, soil available water and welting point after wheat harvest . The data reveal that soil organic matter, soil available water and welting point were significantly improved due to no- tillage system and humic acid application, while soil $\mathrm{pH}$ and bulk density were enhanced only under humic acid treatments. On the other hand, soil salinity did not respond to any of studied treatments . It is obvious to mentioned that increasing humic acid levels increased its effect on soil properties .Also, it worthy to notice that no - tillage had a slightly insignificant effect on increasing soil bulk density. The soil properties and water relations did not respond to the interaction between treatments .

Table (1) .Some soil properties and water relations as affected by tillage system , ,number of irrigations and humic acid .

\begin{tabular}{|c|c|c|c|c|c|c|c|c|c|c|c|c|}
\hline \multirow{2}{*}{ 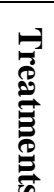 } & \multicolumn{2}{|c|}{ H } & \multicolumn{2}{|c|}{ EC } & \multicolumn{2}{|c|}{ O.M } & \multicolumn{2}{|c|}{$\begin{array}{c}\text { B.D } \\
\left(\mathrm{g} \mathrm{cm}^{-3}\right)\end{array}$} & \multicolumn{2}{|c|}{$\begin{array}{c}\text { Soil available } \\
\text { water(\%) }\end{array}$} & \multicolumn{2}{|c|}{$\begin{array}{c}\text { Wilting point } \\
(\%)\end{array}$} \\
\hline & $\begin{array}{l}1^{\text {st }} \\
8.0\end{array}$ & $\begin{array}{l}2^{\text {nd }} \\
8.1\end{array}$ & $\begin{array}{c}1^{\mathrm{st}} \\
1.23\end{array}$ & $\begin{array}{l}2^{\text {nd }} \\
1.35\end{array}$ & $\begin{array}{c}1^{\mathrm{st}} \\
2.23\end{array}$ & $\begin{array}{c}2^{\text {nd }} \\
2.36\end{array}$ & $\begin{array}{c}1^{\mathrm{st}} \\
1.22\end{array}$ & $\begin{array}{c}2^{\text {nd }} \\
1.20\end{array}$ & $\begin{array}{c}1^{\mathrm{st}} \\
22.57\end{array}$ & $\begin{array}{c}2^{\text {nd }} \\
22.26\end{array}$ & $\begin{array}{c}1^{\text {st }} \\
20.40\end{array}$ & $\begin{array}{c}2^{\text {nd }} \\
20.11\end{array}$ \\
\hline \multicolumn{13}{|c|}{ Main effect of tillage system (A) } \\
\hline $\mathrm{T}_{1}$ & 7.69 & 7.69 & 1.21 & 1.33 & $2.28^{\mathrm{a}}$ & $2.49^{\mathrm{a}}$ & $1.25^{\mathrm{a}}$ & $1.23^{\mathrm{a}}$ & $22.53^{\mathrm{a}}$ & $22.19^{\mathrm{a}}$ & $20.33^{b}$ & $20.21^{\mathrm{b}}$ \\
\hline $\mathrm{T}_{2}$ & 7.59 & 7.59 & 1.22 & 1.13 & $2.14^{\mathrm{b}}$ & $2.36^{\mathrm{b}}$ & $1.21^{\mathrm{b}}$ & $1.19^{\mathrm{b}}$ & 20. & $19.89^{\mathrm{b}}$ & 22.5 & $22.51^{\mathrm{a}}$ \\
\hline \multicolumn{13}{|c|}{ Main effect of irrigation number (B) } \\
\hline $\mathrm{I}_{1}$ & 7.96 & 7.95 & 1.22 & 1.31 & 2.21 & 2.41 & 1.22 & 1.20 & 21 & 21.05 & 21. & 21.35 \\
\hline $\mathrm{I}_{2}$ & 7.96 & 7.95 & 1.21 & 1.33 & 2.22 & 2.43 & 1.23 & 1.22 & & 21.03 & 21.4 & 21.37 \\
\hline \multicolumn{13}{|c|}{ Main effect of humic acid (C) : } \\
\hline $\mathrm{H}_{1}$ & $8.02^{\mathrm{c}}$ & $8.01^{\mathrm{c}}$ & 1.21 & 1.33 & $2.10^{\mathrm{c}}$ & $2.33^{\mathrm{c}}$ & $1.20^{\mathrm{c}}$ & $1.19^{\mathrm{c}}$ & $20.59^{\mathrm{c}}$ & $20.08^{\mathrm{c}}$ & $22.27^{\mathrm{a}}$ & $22.32^{\mathrm{a}}$ \\
\hline $\mathrm{H}_{2}$ & $7.95^{\mathrm{b}}$ & $7.95^{\mathrm{b}}$ & 1.22 & 1.32 & $2.21^{\mathrm{b}}$ & $2.42^{\mathrm{b}}$ & $1.23^{\mathrm{b}}$ & $1.21^{\mathrm{b}}$ & $21.10^{\mathrm{b}}$ & $20.85^{\mathrm{b}}$ & $21.76^{b}$ & $21.55^{\mathrm{b}}$ \\
\hline $\mathrm{H}_{2}$ & $7.91^{\mathrm{a}}$ & $7.91^{\mathrm{a}}$ & 1.22 & 1.31 & $2.23^{\mathrm{a}}$ & $2.54^{\mathrm{a}}$ & $1.26^{\mathrm{a}}$ & $1.24^{\mathrm{a}}$ & $22.66^{\mathrm{a}}$ & $22.44^{\mathrm{a}}$ & $21.76^{\mathrm{c}}$ & $21.55^{\mathrm{c}}$ \\
\hline
\end{tabular}

"Means having some letter ont significant differ at $5 \%$ level of probability "

\section{Soil fertility :}

The effect of tillage system ,irrigation number and humic acid on soil fertility, namely, soil available $\mathrm{N}, \mathrm{P}$ and $\mathrm{K}$ are given in Table (2). The data clearly show that soil available $\mathrm{N}, \mathrm{P}$ and $\mathrm{K}$ were affected only by humic acid application, while tillage system or irrigation number did not affect nutrient availability after wheat harvest . Increasing humic acid were gradually increased soil available $\mathrm{N}, \mathrm{P}$ and $\mathrm{K}$. The increment in soil available $\mathrm{N}, \mathrm{P}$ and $\mathrm{K}$ after harvest due to $24 \mathrm{~kg} \mathrm{ha}^{-1}$ humic acid reached to $8.1,22.9,11.0,24.3,6.1$ and $11.8 \%$ comparing with ,without and $10.0 \mathrm{~kg} \mathrm{ha}^{-1}$ in the first season, respectively. Same trends were obtained in the second season. The soil fertility did not affected by the interaction between treatment.

Fayoum J. Agric. Res. \& Dev., Vol. 34, No.2 July, 2020 
Gihan . A. Mohamed et al.,

Table(2). Soil available $N, P$ and $K$ as affected by tillage system, number of irrigations and humic acid .

\begin{tabular}{|c|c|c|c|c|c|c|}
\hline \multirow{2}{*}{ Treatment } & $\mathbf{N}\left(\mathbf{u g ~ ~ ^ { - 1 }}\right)$ & $\mathbf{P}\left(\mathbf{u g ~ ~ ^ { - 1 }}\right)$ & \multicolumn{2}{c|}{$\left.\mathbf{K ~ U g ~}^{\mathbf{- 1}}\right)$} \\
\cline { 2 - 6 } & $\mathbf{1}^{\text {st }}$ & $\mathbf{2}^{\text {nd }}$ & $\mathbf{1}^{\text {st }}$ & $\mathbf{2 n d}$ & $\mathbf{1}^{\text {st }}$ & $\mathbf{2}^{\text {nd }}$ \\
& $\mathbf{2 3 . 2 5}$ & $\mathbf{2 1 . 1 0}$ & $\mathbf{1 5 . 2}$ & $\mathbf{1 6 . 7}$ & $\mathbf{1 8 5}$ & $\mathbf{1 9 2}$ \\
\hline \multicolumn{7}{|c|}{ The main effect of tillage system (A) } \\
\hline $\mathrm{T}_{1}$ & 23.89 & 21.63 & 18.65 & 15.15 & 189.92 & 177.35 \\
$\mathrm{~T}_{2}$ & 24.09 & 21.90 & 18.55 & 15.15 & 190.25 & 178.57 \\
\hline \multicolumn{7}{|c|}{ The main effect of irrigation number (B) } \\
\hline $\mathrm{I}_{1}$ & 23.98 & 21.82 & 18.57 & 15.13 & 190.05 & 178.02 \\
$\mathrm{I}_{2}$ & 24.08 & 21.72 & 18.63 & 15.17 & 190.17 & 177.90 \\
\hline \multicolumn{7}{|c|}{ The main effect of humic acid (C) } \\
\hline $\mathrm{H}_{1}$ & $21.34^{\mathrm{c}}$ & $19.55^{\mathrm{c}}$ & $16.60^{\mathrm{c}}$ & 15.15 & $179.85^{\mathrm{c}}$ & $165.38^{\mathrm{c}}$ \\
$\mathrm{H}_{2}$ & $24.35^{\mathrm{b}}$ & $21.50^{\mathrm{b}}$ & $18.58^{\mathrm{b}}$ & 17.13 & $189.45^{\mathrm{b}}$ & $177.48^{\mathrm{b}}$ \\
$\mathrm{H}_{3}$ & $26.33^{\mathrm{a}}$ & $24.25^{\mathrm{a}}$ & $20.64^{\mathrm{a}}$ & 19.18 & $201.02^{\mathrm{a}}$ & $191.03^{\mathrm{a}}$ \\
\hline
\end{tabular}

Growth and yield component parameters:

The effect of growth parameters, i.e. plant height, dry weight plant $^{-1}$ and number of tillers plant ${ }^{-1}$ and yield components parameters, namely, number of spikes $\mathrm{m}^{-2}$,number of grains spike and 1000 - grain weight as affected by tillage , irrigation and humic acid treatments are given in Tables ( $3 a, b)$ and $(4 a, b)$. The data show that all studied growth and yield component parameters, except seed weight are higher under (CT) than (NT) system . The increment of these parameters , except 1000 - grain weight due to (CT) system reached to $1.8,6.2,4.8,3.8$ and $5.0 \%$ over (NT) system, respectively in the first season. The corresponding increasing in the second season were 2.5, 8.3, 4.2, 4.5 and $5.5 \%$ in the abovementioned respect. As for irrigation number, the data clearly indicate that irrigated wheat plant five irrigation significantly increased all studied growth and yield component parameters when compared to four irrigations . These treatment increases were 1.4,0.3,1.6,1.7,0.9 and 7.0 \% , respectively in the first season. Some trend were obtained in the second season. With respect to humic acid, the results reveal that, the humic acid application had a positive effect on these parameters, except seed weight. Increasing humic acid up to $24 \mathrm{~kg} \mathrm{ha}^{-1}$ humic acid increased these parameters by about 3.0, $20.8,4.2,5.0$ and $9.0 \%$ over without humic acid in the first season, respectively. Similar trends were obtained in the first season . Considering the effect of the interaction between treatments, the data show that growth and yield component parameters, except seed weight were affected . only by three way interaction among the three factors (AXBXC), where four irrigation under no tillage and $24 \mathrm{~kg}$ ha-1humic acid gave growth and yield component parameters, statistically equal to five irrigation. In general, the highest values of these parameters were achieved under the treatment of (CT ) + five irrigation $+24 \mathrm{~kg}$

Fayoum J. Agric. Res. \& Dev., Vol. 34, No.2 July, 2020 
EFFICIENCY OF HUMIC ACID ON WHEAT PRODUCTIVITY .71 ha-1, or (CT) or (NT) +5 or 4 irrigation $+24 \mathrm{~kg} \mathrm{ha}^{-1}$ humic acid . However , the treatment of (NT) +4 irrigation + no humic acid exhibited the lowest ones .

Table(3.a)Plant height, dry weight and number of tiller splant ${ }^{-1}$ at 75 days age as affected by tillage system, irrigation number and humic acid .

\begin{tabular}{|c|c|c|c|c|c|c|}
\hline \multirow[t]{2}{*}{ Treatments } & \multicolumn{2}{|c|}{ Plant height (cm) } & \multicolumn{2}{|c|}{ Dry weight plant $^{-1}(\mathrm{~g})$} & \multicolumn{2}{|c|}{ Number of tillers plant $^{-1}$} \\
\hline & $1^{\mathrm{st}}$ & $2^{\text {nd }}$ & $1^{\mathrm{st}}$ & $2^{\text {nd }}$ & $1^{\text {st }}$ & $2^{\text {nd }}$ \\
\hline \multicolumn{7}{|c|}{ The main effect of tillage system (A) } \\
\hline$(\mathrm{NT}) \mathrm{T}_{1}$ & $31.02^{\mathrm{b}}$ & $30.57^{\mathrm{b}}$ & $1.95^{\mathrm{b}}$ & $1.92^{\mathrm{b}}$ & $3.10^{\mathrm{b}}$ & $3.07^{\mathrm{b}}$ \\
\hline (CT) $\mathrm{T}_{2}$ & $31.58^{\mathrm{a}}$ & $31.32^{\mathrm{a}}$ & $2.07^{\mathrm{a}}$ & $2.08^{\mathrm{a}}$ & $3.25^{\mathrm{a}}$ & $3.20^{\mathrm{a}}$ \\
\hline \multicolumn{7}{|c|}{ The main effect of number of irrigation $(\mathrm{B})$ : } \\
\hline $\mathrm{I}_{1}$ & $31.08^{\mathrm{b}}$ & $30.79^{b}$ & $1.98^{\mathrm{b}}$ & $1.96^{\mathrm{b}}$ & $3.15^{\mathrm{b}}$ & $3.12^{\mathrm{b}}$ \\
\hline $\mathrm{I}_{2}$ & $31.52^{\mathrm{a}}$ & $31.10^{\mathrm{a}}$ & $2.04^{\mathrm{a}}$ & $2.04^{\mathrm{a}}$ & $3.20^{\mathrm{a}}$ & $3.16^{\mathrm{a}}$ \\
\hline \multicolumn{7}{|c|}{ The main effect of humic acid (C): } \\
\hline $\mathrm{H}_{1}$ & $30.83^{\mathrm{c}}$ & $30.45^{\mathrm{c}}$ & $1.83^{\mathrm{c}}$ & $1.78^{\mathrm{c}}$ & $3.11^{\mathrm{c}}$ & $3.09^{\mathrm{c}}$ \\
\hline $\mathrm{H}_{2}$ & $31.35^{\mathrm{b}}$ & $30.93^{\mathrm{b}}$ & $2.00^{\mathrm{b}}$ & $1.99^{\mathrm{b}}$ & $3.18^{\mathrm{b}}$ & $3.13^{\mathrm{b}}$ \\
\hline $\mathrm{H}_{3}$ & $31.75^{\mathrm{a}}$ & $31.46^{\mathrm{a}}$ & $2.21^{\mathrm{a}}$ & $2.24^{\mathrm{a}}$ & $3.24^{\mathrm{a}}$ & 3.20 \\
\hline
\end{tabular}

"Means having some letter ont significant differ at $5 \%$ level of probability

Table (3.b) Plant height, dry weight /plant and number of tillers /plant ${ }^{-1}$ at 75 days age as affected by three way interaction.

\begin{tabular}{|c|c|c|c|c|c|c|}
\hline \multirow[b]{2}{*}{ Treatments } & \multicolumn{2}{|c|}{ Plant height(cm) } & \multicolumn{2}{|c|}{ Dry weight-1 (g) } & \multicolumn{2}{|c|}{ Number of tillers plant } \\
\hline & $1^{\mathrm{st}}$ & $2^{\text {nd }}$ & $1^{\mathrm{st}}$ & $2^{\text {nd }}$ & $1^{\mathrm{st}}$ & $2^{\text {nd }}$ \\
\hline $\mathrm{T}_{1} \mathrm{XI}_{1} \times \mathrm{H}_{1}$ & $30.2^{\mathrm{d}}$ & $29.9^{\mathrm{d}}$ & 1.72 & $1.69^{\mathrm{e}}$ & $3.01^{\mathrm{f}}$ & 3.00 \\
\hline $\mathrm{T}_{1} \mathrm{XI}_{1} \times \mathrm{H}_{2}$ & $30.7^{\mathrm{cd}}$ & $30.5^{\mathrm{c}}$ & 1.89 & $1.85^{\mathrm{cd}}$ & $3.08^{\mathrm{e}}$ & $3.05^{c}$ \\
\hline $\mathrm{T}_{1} \times \mathrm{I}_{1} \times \mathrm{XH}_{3}$ & $31.3^{\mathrm{b}}$ & $31.2^{\mathrm{b}}$ & 2.15 & $2.15^{\mathrm{b}}$ & $3.26^{\mathrm{b}}$ & $3.23^{\circ}$ \\
\hline $\mathrm{T}_{1} \mathrm{XI}_{2} \mathrm{XH}_{1}$ & $30.8^{\mathrm{bc}}$ & $30.5^{\mathrm{c}}$ & 1.82 & $1.78^{\mathrm{d}}$ & $3.06^{\mathrm{e}}$ & 3.04 \\
\hline $\mathrm{T}_{1} \mathrm{XI}_{2} \mathrm{XH}_{2}$ & $31.4^{\mathrm{ab}}$ & $31.2^{\mathrm{b}}$ & 1.94 & $1.90^{\mathrm{cd}}$ & $3.12^{\mathrm{de}}$ & 3.09 \\
\hline $\mathrm{T}_{1} \mathrm{XI}_{2} \mathrm{XH}_{3}$ & $31.7^{\mathrm{ab}}$ & $31.6^{\mathrm{ab}}$ & 2.16 & $2.16^{\mathrm{b}}$ & $3.28^{\mathrm{ab}}$ & 3.23 \\
\hline $\mathrm{T}_{2} \mathrm{XI}_{1} \mathrm{XH}_{1}$ & $30.9^{\mathrm{bc}}$ & $30.8^{\mathrm{bc}}$ & 1.85 & $1.81^{\mathrm{d}}$ & $3.14^{\mathrm{d}}$ & 3.12 \\
\hline $\mathrm{T}_{2} \mathrm{XI}_{1} \mathrm{XH}_{2}$ & $31.5^{\mathrm{ab}}$ & $31.4^{\mathrm{b}}$ & 1.99 & $1.94^{\mathrm{c}}$ & $3.20^{\mathrm{c}}$ & $3.15^{\mathrm{b}}$ \\
\hline $\mathrm{T}_{2} \mathrm{XI}_{1} \mathrm{XH}_{3}$ & $31.9^{\mathrm{a}}$ & $31.9^{\mathrm{a}}$ & 2.25 & $2.31^{\mathrm{a}}$ & $3.31^{\mathrm{a}}$ & 3.26 \\
\hline $\mathrm{T}_{2} \mathrm{XI}_{2} \mathrm{XH}_{1}$ & $31.4^{\mathrm{ab}}$ & $31.1^{\mathrm{b}}$ & 1.91 & $1.84^{\mathrm{cd}}$ & $3.21^{\mathrm{c}}$ & $3.18^{\mathrm{l}}-\ln$ \\
\hline $\mathrm{T}_{2} \mathrm{XI}_{2} \mathrm{XH}_{2}$ & $31.8^{\mathrm{ab}}$ & $31.6^{\mathrm{ab}}$ & 2.15 & $2.24^{\mathrm{ab}}$ & $3.29^{\mathrm{ab}}$ & 3.29 \\
\hline $\mathrm{T}_{2} \mathrm{XI}_{2} \mathrm{xH}_{3}$ & $32.0^{\mathrm{a}}$ & $31.8^{\mathrm{ab}}$ & 2.26 & $2.31^{\mathrm{a}}$ & $3.32^{\mathrm{a}}$ & 3.26 \\
\hline
\end{tabular}

"Means having some letter ont significant differ at $5 \%$ level of probability

Fayoum J. Agric. Res. \& Dev., Vol. 34, No.2 July, 2020 
Gihan . A. Mohamed et al.,

Table(4.a) Number of spikes, number of grains spike ${ }^{-1}$ and1000- grain weight as affected by tillage system, irrigation number and humic acid .

\begin{tabular}{|c|c|c|c|c|c|c|}
\hline \multirow{2}{*}{ Treatments } & Number of spikes $\left.\mathbf{m}^{-2}\right)$ & \multicolumn{2}{c|}{ grains spike $^{-1}$} & \multicolumn{1}{c|}{ 1000- grain weight } \\
\cline { 2 - 7 } & $1^{\text {st }}$ & $2^{\text {nd }}$ & $1^{\text {st }}$ & $2^{\text {nd }}$ & $1^{\text {st }}$ & $2^{\text {nd }}$ \\
\hline \multicolumn{7}{|c|}{ The main effect of tillage system $(\mathrm{A}):$} \\
\hline (NT) $\mathrm{T}_{1}$ & $419.0^{\mathrm{b}}$ & $410.6^{\mathrm{b}}$ & $40.2^{\mathrm{b}}$ & $39.8^{\mathrm{b}}$ & 54.8 & 54.60 \\
$(\mathrm{CT}) \mathrm{T}_{2}$ & $431.0^{\mathrm{a}}$ & $425.3^{\mathrm{a}}$ & $40.0^{\mathrm{a}}$ & $41.7^{\mathrm{a}}$ & 54.75 & 54.60 \\
\hline \multicolumn{7}{|c|}{} \\
\hline $\mathrm{I}_{1}$ & $419.3^{\mathrm{b}}$ & $413.06^{\mathrm{b}}$ & $1.98^{\mathrm{b}}$ & $40.4^{\mathrm{b}}$ & $53.20^{\mathrm{b}}$ & 53.1 \\
$\mathrm{I}_{2}$ & $430.6^{\mathrm{c}}$ & $424.4^{\mathrm{a}}$ & $2.04^{\mathrm{a}}$ & $41.1^{\mathrm{a}}$ & $56.40^{\mathrm{a}}$ & 65.2 \\
\hline \multicolumn{7}{|c}{} \\
\hline $\mathrm{H}_{1}$ & $407.3^{\mathrm{c}}$ & $401.2^{\mathrm{c}}$ & $39.3^{\mathrm{c}}$ & $39.1^{\mathrm{c}}$ & 54.8 & 54.6 \\
$\mathrm{H}_{2}$ & $428.9^{\mathrm{b}}$ & $422.2^{\mathrm{b}}$ & $41.1^{\mathrm{b}}$ & $40.8^{\mathrm{b}}$ & 54.8 & 54.7 \\
$\mathrm{H}_{3}$ & $438.7^{\mathrm{a}}$ & $431.9^{\mathrm{a}}$ & $42.8^{\mathrm{a}}$ & $42.8^{\mathrm{a}}$ & 54.8 & 54.7 \\
\hline
\end{tabular}

"Means having some letter ont significant differ at $5 \%$ level of probability

Table(4.b) Number of spikes and number of grains spike ${ }^{-1}$ as affected by three way interaction.

\begin{tabular}{|c|c|c|c|c|}
\hline \multirow[t]{2}{*}{ Treatments } & \multicolumn{2}{|c|}{ Number of spikes $(\mathrm{cm})$} & \multicolumn{2}{|c|}{ Grains spike $^{1}$} \\
\hline & $1^{\mathrm{st}}$ & $2^{\text {nd }}$ & $\mathbf{1}^{\text {st }}$ & $2^{\text {nd }}$ \\
\hline $\mathrm{T}_{1} \times \mathrm{II}_{1} \times \mathrm{H}_{1}$ & $382.6^{\mathrm{g}}$ & $373.5^{\mathrm{g}}$ & $37.1^{\mathrm{e}}$ & $36.9^{f}$ \\
\hline $\mathrm{T}_{1} \mathrm{XI}_{1} \times \mathrm{H}_{2}$ & $415.1^{\mathrm{e}}$ & $406.1^{\mathrm{e}}$ & $39.2^{\mathrm{d}}$ & $39.0^{\mathrm{e}}$ \\
\hline $\mathrm{T}_{1} \times \mathrm{I}_{1} \times \mathrm{H}_{3}$ & $436.2^{\mathrm{f}}$ & $430.1^{\mathrm{ab}}$ & $42.8^{\mathrm{ab}}$ & $42.6^{\mathrm{ab}}$ \\
\hline $\mathrm{T}_{1} \mathrm{xI}_{2} \times \mathrm{H}_{1}$ & $409.6^{\mathrm{f}}$ & $401.2^{\mathrm{f}}$ & $39.3^{\mathrm{d}}$ & $39.0^{\mathrm{e}}$ \\
\hline $\mathrm{T}_{1} \mathrm{xI}_{2} \times \mathrm{H}_{2}$ & $430.6^{c}$ & $423.3^{\mathrm{cd}}$ & $41.0^{\mathrm{c}}$ & $39.7^{\text {cde }}$ \\
\hline $\mathrm{T}_{1} \mathrm{xI}_{2} \times \mathrm{H}_{3}$ & $439.6^{\mathrm{a}}$ & $429.0^{\mathrm{b}}$ & $41.9^{b}$ & $41.6^{\mathrm{bc}}$ \\
\hline $\mathrm{T}_{2} \times \mathrm{I}_{1} \times \mathrm{H}_{1}$ & $410.2^{f}$ & $407.6^{\mathrm{e}}$ & $39.5^{\mathrm{d}}$ & $39.3^{\text {de }}$ \\
\hline $\mathrm{T}_{2} \times \mathrm{I}_{1} \times \mathrm{H}_{2}$ & $432.5^{\mathrm{c}}$ & $428.6^{\mathrm{b}}$ & $41.6^{\mathrm{b}}$ & $41.5^{\mathrm{bc}}$ \\
\hline $\mathrm{T}_{2} \times \mathrm{I}_{1} \times \mathrm{H}_{3}$ & $439.3^{\mathrm{ab}}$ & $431.3^{\mathrm{ab}}$ & $43.6^{\mathrm{a}}$ & $43.3^{\mathrm{a}}$ \\
\hline $\mathrm{T}_{2} \times \mathrm{I}_{2} \times \mathrm{H}_{1}$ & $426.6^{\mathrm{d}}$ & $422.5^{\mathrm{d}}$ & $41.3^{\mathrm{c}}$ & $41.0^{c}$ \\
\hline $\mathrm{T}_{2} \mathrm{XI}_{2} \times \mathrm{H}_{2}$ & $437.5^{\mathrm{ab}}$ & $430.9^{\mathrm{ab}}$ & $42.7^{\mathrm{ab}}$ & $42.4^{\mathrm{ab}}$ \\
\hline $\mathrm{T}_{2} \mathrm{XI}_{2} \times \mathrm{H}_{3}$ & $439.8^{\mathrm{a}}$ & $432.6^{\mathrm{a}}$ & $43.0^{\mathrm{a}}$ & $42.9^{\mathrm{a}}$ \\
\hline
\end{tabular}

"Means having some letter ont significant differ at $5 \%$ level of probability

\section{Yield parameters:}

The data in Table (5 a,b) show the main effect of tillage system, irrigation number and humic acid as well as their interaction on grain, straw and biological yields of wheat. The data clearly show that all studied yield parameter were significantly affected by the studied three factors. The conventional tillage (CT) exhibited the highest wheat yields than no tillage system. The relative increasing in grain, straw and biological yields due to (CT) over (NT) system were 8.7,8.7 and 8.7

Fayoum J. Agric. Res. \& Dev., Vol. 34, No.2 July, 2020 
EFFICIENCY OF HUMIC ACID ON WHEAT PRODUCTIVITY 73

$\%$ in the first season ; and 11.2,8.2 and $10.0 \%$ in the second one. Watered wheat plants five irrigations gave higher grain, straw and biological yields than four irrigations in both seasons. As for humic acid, the data show that increasing the level of humic acid resulted in significantly increasing in wheat yields . Added with $24 \mathrm{~kg}$ ha $^{-1}$ humic acid increased grain, straw and biological yields by about 3.5 and 8.7, 5.2 and 12.1 , and 4.6 and $10.9 \%$ than 10 and $0.0 \mathrm{~kg} \mathrm{ha}^{-1}$ humic acid in first season . Same trends were obtained in the second season. With respect to the interaction effect, the results in Table (5) show that wheat yields were responded only to the three way interaction $(\mathrm{AxBxC})$, where wheat plants supplied $24 \mathrm{~kg}$ humic acid ha ${ }^{-1}$ and irrigated four irrigations under no tillage system produced wheat yields, statistically equal to those under (CT) system, five irrigations and full dose of humic acid . In general, the highest wheat yields were obtained under the treatments of (CT) system + five irrigation $+24 \mathrm{~kg} \mathrm{ha}^{-1}$ humic acid or (NT) + four irrigation +24 $\mathrm{kg} \mathrm{ha}^{-1}$ humic acid. On the other hand, the lowest wheat yields were recorded under the treatment of (NT) system + four irrigation + without humic .

Table(5.a) Grain, straw and biological yields as affected by tillage system, irrigation number and humic acid .

\begin{tabular}{|c|c|c|c|c|c|c|}
\hline \multirow[t]{2}{*}{ Treatments } & \multicolumn{2}{|c|}{ Grain yield $\left(\mathrm{t} \mathrm{ha}^{-1}\right)$} & \multicolumn{2}{|c|}{ Straw yield $\left(\mathrm{t} \mathrm{ha}{ }^{-1}\right)$} & \multicolumn{2}{|c|}{ Biological yield $\left(\mathrm{t} \mathrm{ha}^{-1}\right)$} \\
\hline & $\mathbf{1}^{\text {st }}$ & $2^{\text {nd }}$ & $1^{\text {st }}$ & $2^{\text {nd }}$ & $1^{\text {st }}$ & $2^{\text {nd }}$ \\
\hline \multicolumn{7}{|c|}{ The main effect of tillage system (A): } \\
\hline $\mathrm{T}_{1}$ & $8.90^{\mathrm{b}}$ & $8.77^{\mathrm{b}}$ & $15.92^{\mathrm{b}}$ & $16.03^{\mathrm{b}}$ & $24.82^{b}$ & $24.80^{\mathrm{b}}$ \\
\hline $\mathrm{T}_{2}$ & $9.67^{\mathrm{a}}$ & $9.75^{\mathrm{a}}$ & $17.30^{\mathrm{a}}$ & $17.35^{\mathrm{a}}$ & $26.97^{\mathrm{a}}$ & $27.28^{\mathrm{a}}$ \\
\hline \multicolumn{7}{|c|}{ The main effect of number of irrigation $(\mathrm{B})$ : } \\
\hline $\mathrm{I}_{1}$ & $9.20^{\mathrm{b}}$ & $9.08^{b}$ & $16.40^{\mathrm{b}}$ & $16.50^{\mathrm{b}}$ & $25.60^{\mathrm{b}}$ & $25.76^{\mathrm{b}}$ \\
\hline $\mathrm{I}_{2}$ & $9.56^{\mathrm{c}}$ & $9.34^{\mathrm{a}}$ & $16.93^{\mathrm{a}}$ & $16.88^{\mathrm{a}}$ & $26.36^{\mathrm{a}}$ & $26.05^{\mathrm{a}}$ \\
\hline \multicolumn{7}{|c|}{ The main effect of humic acid (C): } \\
\hline $\mathrm{H}_{1}$ & $8.88^{c}$ & $8.56^{c}$ & $15.64^{c}$ & $15.75^{\mathrm{c}}$ & $24.52^{c}$ & $24.53^{c}$ \\
\hline $\mathrm{H}_{2}$ & $9.32^{b}$ & $9.20^{b}$ & $16.67^{b}$ & $16.88^{b}$ & $25.99^{\mathrm{b}}$ & $26.08^{b}$ \\
\hline $\mathrm{H}_{3}$ & $9.65^{\mathrm{a}}$ & $10.08^{a}$ & $17.54^{\mathrm{a}}$ & $17.45^{\mathrm{a}}$ & $27.19^{\mathrm{a}}$ & $27.53^{\mathrm{a}}$ \\
\hline
\end{tabular}

Fayoum J. Agric. Res. \& Dev., Vol. 34, No.2 July, 2020 
Gihan . A. Mohamed et al.,

Table(5.b) Grain,straw and biological yields as affected by the three way interaction .

\begin{tabular}{|c|c|c|c|c|c|c|}
\hline \multirow[b]{2}{*}{ Treatments } & \multicolumn{2}{|c|}{ Grain yield (t ha ${ }^{-1}$ ) } & \multicolumn{2}{|c|}{ Straw yield $\left(\mathrm{t} \mathrm{ha}^{-1}\right)$} & \multicolumn{2}{|c|}{ Biological yield $\left(\mathrm{t} \mathrm{ha}^{-1}\right)$} \\
\hline & $1^{\text {st }}$ & $2^{\text {nd }}$ & $1^{\mathrm{st}}$ & $2^{\text {nd }}$ & $1^{\text {st }}$ & $2^{\text {nd }}$ \\
\hline $\mathrm{T}_{1} \mathrm{XI}_{1} \times \mathrm{H}_{1}$ & $7.68^{f}$ & $7.55^{\mathrm{f}}$ & $14.21^{\mathrm{e}}$ & $14.30^{\mathrm{d}}$ & $21.89^{\mathrm{d}}$ & $21.85^{\mathrm{d}}$ \\
\hline $\mathrm{T}_{1} \times \mathrm{I}_{1} \times \mathrm{H}_{2}$ & $8.85^{\mathrm{e}}$ & $8.73^{\mathrm{e}}$ & $15.67^{\mathrm{c}}$ & $15.70^{\mathrm{bc}}$ & $24.52^{b c}$ & $24.43^{c}$ \\
\hline $\mathrm{T}_{1} \times \mathrm{I}_{1} \times \mathrm{H}_{3}$ & $9.65^{\mathrm{ab}}$ & $9.52^{b c}$ & $17.10^{\mathrm{ab}}$ & $17.10^{\mathrm{ab}}$ & $26.75^{\mathrm{ab}}$ & $26.62^{\mathrm{ab}}$ \\
\hline $\mathrm{T}_{1} \mathrm{XI}_{2} \mathrm{xH}_{1}$ & $8.63^{\mathrm{e}}$ & $8.51^{\mathrm{e}}$ & $15.31^{\mathrm{d}}$ & $15.40^{\mathrm{c}}$ & $23.94^{\mathrm{c}}$ & $23.91^{\mathrm{c}}$ \\
\hline $\mathrm{T}_{1} \times \mathrm{I}_{2} \times \mathrm{H}_{2}$ & $8.93^{\mathrm{e}}$ & $8.77^{\mathrm{de}}$ & $16.12^{\mathrm{c}}$ & $16.40^{\mathrm{bc}}$ & $25.05^{\mathrm{bc}}$ & $25.17^{\mathrm{ab}}$ \\
\hline $\mathrm{T}_{1} \mathrm{XI}_{2} \mathrm{XH}_{3}$ & $9.65^{\mathrm{ab}}$ & $9.52^{b c}$ & $17.12^{\mathrm{ab}}$ & $17.30^{\mathrm{ab}}$ & $26.77^{\mathrm{ab}}$ & $26.82^{\mathrm{bc}}$ \\
\hline $\mathrm{T}_{2} \times \mathrm{I}_{1} \times \mathrm{H}_{1}$ & $9.87^{\mathrm{a}}$ & $8.67^{\mathrm{e}}$ & $16.20^{\mathrm{bc}}$ & $16.60^{b}$ & $26.07^{\mathrm{b}}$ & $25.27^{\mathrm{ab}}$ \\
\hline $\mathrm{T}_{2} \times \mathrm{I}_{1} \times \mathrm{H}_{2}$ & $9.51^{b}$ & $9.39^{c}$ & $17.25^{\mathrm{ab}}$ & $17.80^{\mathrm{a}}$ & $26.76^{\mathrm{ab}}$ & $27.19^{\mathrm{ab}}$ \\
\hline $\mathrm{T}_{2} \times \mathrm{I}_{1} \times \mathrm{H}_{3}$ & $9.65^{\mathrm{ab}}$ & $10.62^{\mathrm{a}}$ & $17.95^{\mathrm{a}}$ & $17.50^{\mathrm{ab}}$ & $27.60^{\mathrm{a}}$ & $28.12^{\mathrm{a}}$ \\
\hline $\mathrm{T}_{2} \mathrm{XI}_{2} \times \mathrm{H}_{1}$ & $9.33^{b}$ & $9.28^{\mathrm{c}}$ & $16.83^{b}$ & $16.70^{b}$ & $26.16^{\mathrm{b}}$ & $25.98^{b}$ \\
\hline $\mathrm{T}_{2} \times \mathrm{I}_{2} \times \mathrm{H}_{2}$ & $9.98^{\mathrm{a}}$ & $9.90^{b}$ & $17.62^{\mathrm{ab}}$ & $17.60^{\mathrm{ab}}$ & $27.60^{\mathrm{a}}$ & $27.50^{\mathrm{a}}$ \\
\hline $\mathrm{T}_{2} \mathrm{XI}_{2} \times \mathrm{H}_{3}$ & $9.75^{\mathrm{ab}}$ & $10.63^{\mathrm{a}}$ & $17.96^{\mathrm{a}}$ & $17.90^{\mathrm{a}}$ & $27.61^{\mathrm{a}}$ & $28.53^{\mathrm{a}}$ \\
\hline
\end{tabular}

Means having some letter ont significant differ at $5 \%$ level of probability

\section{DISCUSSION}

The positive effect of minimizing tillage system on limiting soil erosion is well known, beside that it improving chemical, physical and biological properties as well as increasing soil organic matter which in turn enhanced crop productivity (Willan , 1997 ). The data of this research show that reducing tillage process from convential tillage (CT) to no tillage (NT) were not significantly affected soil reaction and soil salinity as well as soil available N,P and K after wheat harvest . On the other hand ,(NT) system increased soil organic matter and bulk density as well as improved water relations in term of soil available water and wilting point, which it increase soil available water and reduce the wilting point than (CT) system . In this connection, Moraru and Rusu (2012) mentioned that no tillage reduce soil mobilization, therefore soil became compacted which directly related to soil type and its state of degradation, accordingly increased organic matter and bulk density.

They added, no tillage system caused more significantly penetration resistance, hence improved water relations. These results are in line with those obtained by Martins et al (2011) and Russa et al (2011). Adversely, convential tillage system had positive effect on wheat growth, yield and its components than no tillage, which mainly due to high penetration resistance in no tillage system reduced root growth (Moraru and Rusu , 2010, 2011 ). Also, Mekky et al (2007) reported that the increase in wheat growth and yield component due to convential tillage may be attributed to either to weed control by increasing tillage or improving soil porosity . Similar results were obtained by Arora et al (1991) and Memon et al (2013).

Fayoum J. Agric. Res. \& Dev., Vol. 34, No.2 July, 2020 
EFFICIENCY OF HUMIC ACID ON WHEAT PRODUCTIVITY 75

It is evident from the results that the number of irrigations did not significantly affect soil properties and fertility after wheat harvest. However, it had positive effect on vegetative growth and yield and its components of wheat, where watered wheat plants five irrigations exhibited the highest values than four one. The increase in plant height may be attributed to the increase in nutrient uptake with increasing moisture availability for plant up take (Gangaiah ,2005) . The enhancement of dry weight plant ${ }^{-1}$ under five irrigations is mainly due to its effect on plant height and secondary continuous vertical growth of plant, consequently reflected greater dry matter plant ${ }^{-1}$ (Jat et al 2015). Five irrigation treatment produce highest number of tillers plant ${ }^{-1}$ than four irrigation, which mainly due to more formation of photosynthesis and its translocation to the other organs caused in more formation of tillers in plant (Jat et al 2017). Moreover, reduced irrigation number from five to four irrigations resulted in significant reduction in yield and yield component parameters, namely, number of spikes $\mathrm{m}^{-2}$, number of grains spike ${ }^{-1}$, 1000 - grain weight, grain yield, straw yield and biological yield . The decreasing in these parameters caused by four irrigation, is mainly ascribed to the negative effect of stress conditions on growth parameters as discussed before. In this concern , Abd El- Gayed and Bachandy, (2018) mentioned that, water stress can induce a significant reduction in formation of assimilates by photosynthetic organs . These results of the effect of irrigation treatment on vegetative and yield and yield components of wheat are in line with those obtained by Ahmed et al (2010), Rahim et al (2110), Gupta et al (2012) and Minguming et al (2018) .

On basis of the experimental results, it was found that, humic acid improved all studied soil properties and fertility, except soil salinity which mainly due to humic acid was produced from nitrogen compound, containing decomposed amino acids and organic complex include carboxyl $\left(\mathrm{COOH}^{-1}\right)$ and phenols $\left(\mathrm{OH}^{-1}\right)$ groups , in turn improve soil properties and fertility (Schnitzer, 1992). Mac Carthy et al (2001) stated that humic acid improve soil structure and nutrient uptake . In addition , Bhatti et al (2011) mentioned that humic acid affect plant growth in two ways : indirectly by enhanced physical and chemical and biological soil properties and directly by increases chlorophyll content, improve plant respiration and growth hormones, increases plant membranes penetration .......ect. As for its effect on soil fertility Verlinder et al (2009) stated that humic acid enhance the solubility of nutrient by building complex compounds or chelating with cations . Similar results were obtained by Ismail et al (2014), Ismail et al (2016) and Sarhan and Abd El Gayed (2017) who reported that humic acid improve physical and chemical soil properties as well as soil fertility. Also Wang et al (1995) and Cavusoglu (2017) found that humic increased nutrient availability and decreased soil $\mathrm{pH}$. As for wheat productivity, the data show that wheat growth and yield and its components were significantly responded to humic acid application. The promotive effect of humic acid on wheat growth may be due to its effect on improving lateral root development

Fayoum J. Agric. Res. \& Dev., Vol. 34, No.2 July, 2020 
Gihan . A. Mohamed et al.,

by activating the membrane of cells and $\mathrm{pH}$ pump in tonoplast of plant cell (Zandonddi: et al 2007). In addition, the positive effect of humic acid may be attributed to humic acid results in root development (Baldotto et al (2012 ), enhances the shoots to store nutrients in its leaves (Chen et al ,2004) and it increase nutrient uptake by roots through cell membrane (Yilmaz, 2007). The results are in accordance with those obtained by Ukan (2008), Tahir et al (2011), Ismail et al (2014), Sarhan and Abd El-Gayed(2017) and Bezuglova (2017).

It is evident from the data that growth and yield and yield components of wheat were significantly affected by the three way interaction among the three studied factors (AXBXC) where using the high level of humic acid $\left(\mathrm{H}_{3}\right)$ under no tillage $(\mathrm{CT})$ and moisture stress condition (irrigation four times , $\mathrm{I}_{2}$ ) resulted in wheat productivity statistically equal to those under convential tillage and irrigated five times. This results means the possibility to reduce the cost of tillage process and save one irrigation by added $24 \mathrm{~kg}$ humic acid ha ${ }^{-1}$ as soil application before wheat planting. The promotive effect of humic acid on enhancing the effect of reducing both tillage and irrigation is mainly due to its positive effect on water relation as discussed before (Table3). Similar results were obtained by Shahryar and Mollasedeghi (2011) who reported that humic acid decreased drought stress intensity and increased economic and biological yield of wheat .

\section{CONCLUSSION}

From the results of this study it could be concluded that supplying surface claying soils with $24 \mathrm{~kg} \mathrm{ha}^{-1}$ humic acid reduced tillage process and irrigation numbers from five to four irrigations without any reduction in wheat productivity and improved soil properties and fertility .

\section{REFERENCES}

Abd El-Gayed and S. O. Bashandy (2018) . Effect of reduced irrigation and potassium fertilization on quantity and quality of Giza 95 cotton plants . Egypt.J. Agron.The 15 ${ }^{\text {th }}$ Int .Conf . Crop Science, pp.71-84 .

Ahmad , M., A. Ghafoor ., M. Asif and H.U. Farid (2010) . Effect of irrigation techniques on wheat production and water saving in soils. Soil \& Environ . 29(1) : 69-72.

A.O.A.C.(1990). Official Method OfAnalysis " Association Official Analytical Chemists " $10^{\text {th }}$ Ed., Washington , D.C., USA.

Arora,V.K., P.R.Gajra and S.S.Prihar (1991) .Tillage effect on corn in sandy soil relation to water retentivity, nutrient and water management and seasonal evaporability .Soil and Tillage Res.21 (1) :1-21.

Badotto ,M.A. ,R.C.Muniz and L.B.Dobbss(2011).Root growth of Arabidopsis thaliana (L) Heynh,treated with humic acids isolated from typical soils of Rio de Janeiro State , Brazil, Revista Ceres , 58:504-511.

Fayoum J. Agric. Res. \& Dev., Vol. 34, No.2 July, 2020 
EFFICIENCY OF HUMIC ACID ON WHEAT PRODUCTIVITY 77

Baquedano , F. J and F. J. Castillo (2006). Comparative ecophysiological effect of drought on seedlings of the Mediterranean water saver Pinus halepensis and water -spenders Quercus coccifera and Quercus ilex . Trees , 20 : 689-700 .

Bhatti , M.B., I.Rajpar ,Z.Ul-hassan , A.N. Shah and S.D. Tunio (2011). Humic acid improves growth, yield and oil content of Brassica Compestris L.Pak . J. Agri., Agril. Engg., Vet. Sci., 27(2)125-133 .

Bezuglova,O.S.,E.A.Polienko,A.V.Gorovtsov,V.A.L.yhman and P.D.Pavlov(2017) . The effect ofhumic substances on winter wheat yield and fertility of ordinary chernozem. Annals of Agrarian Science ., 15 :239-242.

Canellas,L.P.,A.L.Olivares , O.A.L.Facanha and A.R.Facanha (2002). Humic acids isolated from earthworm compost enhance $\mathrm{H}^{+}$- ATPase activity in maize roots, Plant Physiology . 30:1951-1957 .

Cavusoglu ,M.N.,N.Turkogluand F.A.Ozdemir(2017) . The effect of phosphorus and humic acid on some soil properties and flowers quality of gladiolus, 19 (1):150-155.

Chapman , H.D.and P.F. Pratt (1691). Methods of Analysis for Soil , Plant and Water. Univ of California, USA.

Chen,Y., M.Nobili and T. Aviad (2004). Stimulatory effect of humic substances on plant growth . In: MAGDOFT.,RAY R.(eds): Soil Organic Matter in Sustainable Agriculture . CRC Press, Washington .

El-Shanhorey , N.A. (2015) . Effect of different levels of irrigation on growth , flowering and chemical constituents of Jatropha Curcas plants grown in sandy soil (2015) . Bull . Fac . Agric., Cario Univ ., 66:130-141 .

Gupta ,S.,V.P. Agrawal , N.K. Gupta (2012) . Efficacy of putrescine and benzyladenine on photosynthesis and productivity in relation to drough tolerance in wheat (Triticum aestivum L.) J., Physiol Mol Biol Plants ,Dol 10.1007/s 12298-012-0123-9.

Hobbs, P. R., K. Sayre and R. gupta (2008). The role of conservation in sustainable agriculture philosophical transactions of the royal society B:Biological , 363: 543-555.

Irshad, S., S. Muhammad, A. Muhammad, A. G. Muhammad and R. b R. Muhammad (2017).Interaction effect of different farmyard manure levels and tillage operations on yield attributes of cotton,Advances in Environmental Biology ,11(5) :44-48.

Ismail, A.S.; A. M. Abd El Hafeez and O. A. M. Galal (2014). Response ofsoybean (Glycine max L.) to microbial inoculation under chemical fertilization: 1- nodulation, yield and its components. Egypt. J. of Appl. Sci.,29 (2B) :1237-1258.

Fayoum J. Agric. Res. \& Dev., Vol. 34, No.2 July, 2020 
Gihan . A. Mohamed et al.,

Ismail ,S.A .,O.A. Galal and M.G,Sarhan (2016) . Effect of foliar micronutrients under some bio-stimulants on the productivity of faba bean (ViciafabaL.).FayoumJ .Agric . Res.\&Dev .,32(1): 71-87.

Jat , R.K , A.G. Patel , A. Shviran and A.L.Bijarnia (2015) . Response of oat (Avena sativa L.) to nitrogen and phosphorus levels under North Gujarat Agro-climatic conditions. Journal of Eco- Friendly Agriculture ., 10 : 3924 .

Jat , H., M.K Kaushik , V . Nepalia and D. Singh (2017) . Effect of irrigation schedule and nitrogen fertilization on growth, yield and quality of fodder oat (Avena Sativa L. ) . Journal of Pharmacognosy and Phytochemistry ,6 (4): 2040- 2042 .

Kozlowski, T.T., S.G. Pallardy (2002) . Acclimation and adaptive response of woody plants to environmental stresses . The Bot ,Rev . 68 (2) : 270334.

Lal , R. , (1990) .Soil erosion in the tropics : principles and management $\mathrm{M}_{\mathrm{c}} \mathrm{Graw}-$ Hill Inc .

Mackowiak, C.L., P.R. Grossi and B.G.Bugbee (2001).Beneficial effects of humic acid on mirconutrient availability to wheat .Soil Science Society of America Journal , 56: 1744-1750 .

Martins,T.,S.C.Saab,D.M.B.P. Milori , A.M.Brinatti , Soil \& Tillage Research ,111: 231-235.

Mekky, M.s., M.A.Ali and S.A. Elmajeed (2007) .Effect of mechanical tillage system, water varieties and weed control treatment on weeds and wheat (Triticum Aestivuml.)productivity . Misr J Ag . Eng., 24 (1): 18-41.

Memon ,S.Q., M.S. Miejat, A.Q.Mughal , A.A. Mirani and H.I.Javed (2013). Tillage and NPK effect on growth and yield of spring maize in Islamabad , Pakistan .J. Agric.Res 26 (1) 32-38.

Ming-ming , Z., D.Baondi , Q.Yun-zhou , S. Chang - hai , Y. Hong , W.Ya-kai and L. Meng-Yu (2018). Yield and water use response of winter wheat to irrigation and nitrogen application in North China Plain . Journal of Integrative Agriculture, 17 (0) : 60345-7 .

Moraru .,P.I and T.Rusu (2012) .Effect of tillage system on soil moisture , soil temperature, soil respiration and production of wheat, maize and soybean crops. Journal of Food, Agriculture \&Environment Vol.10(2):445-448.

Moraru .,P.I and T.Rusu $\left(\mathbf{2 0 1 0}^{\mathrm{a}}\right)$.Soil tillage conservation and its on soil organic matter, water management and carbon sequestration . Journal of Food, Agriculture \&Environment Vol.8(3-4):309-312.

Moraru .,P.I , T.Rusu and M.L. Sopterean $\left(\mathbf{2 0 1 0}^{\mathrm{b}}\right)$.Soil tillage conservation and its on soil organic matter, water management and carbon sequestration . In Pro Environment / Pro Mediu Vol.1,pp.359-366.

Fayoum J. Agric. Res. \& Dev., Vol. 34, No.2 July, 2020 
EFFICIENCY OF HUMIC ACID ON WHEAT PRODUCTIVITY ... 79

Rahim , A., A. M. Ranjha, Rahamtullah and E.A. Waraich (2010). Effect of phosphorus application and irrigation scheduling on wheat yield and phosphorus use efficiency . Soil \& Environ . 29 (1) : 15-22 .

RUSU, T., P.I.Moraru , O.Ranta, I.Drocas , I. Bogdan , A.I.POPand M.L.Sopterean (2011).No-tillage and minimum tillage- their impact on soil compaction, water dynamics, soil temperature and production on wheat, maize and soybean crop. Bulletin UASVM Agriculture, 68(1) :1843-5386.

Salman,S. R., S. D. Abou-hussein , A. M. R.Abdel - Mawgoud and A.El-Nemr (2005). Furit yield and quality of watermelon as affected by hybrids and humic acid application. Journalof Applied Science Research 1 (1) :51-58.

Sarhan , M.G.R. and S.Sh. Abd El- Gayed (2017). The possibility of using feldspar as alernatine potassium for cotton fertilization in combined with silicate dissolving bacteria, humic acid and farmyard manure and its effect on soil properties .J., Soil Sci and Agric . Eng ,Mansoura Univ, Vol 8 (12) : 761-767.

Schnitzer,M.(1992).Significance of soil organic matter in soil formation ,transport processes in soil and in the formation of soil structure .Soil Utilization and Soil Fertility Volume 4 Humus Budget ,206,63-81 .

Shahryari , R. and V. Mollasdeghi (2011). Harvest index and its as associated characters in winter wheat genotypes against terminal drought at presence of a peat derived humic fertilizer. Advances in Environmental Biology, 5(1) : 162-165.

Tebrugge ,F. and R.A.During (1999). Reducing tillage intensity -a review of results from a long - term study in Germany .Soil and Tillage Research $, 53: 15-28$.

Tahir , M.M., M. Khurshid , M.Z. Khan , M.K. Abbasi and M.H. Kazmi (2011) . Lignite- derived humic acid effect on growth of wheat plants in different soils . Soil Sci . Soc. Of China, pedosphere , 21 (1) : 124-131

Vakali ,C., J.G.Zaller and U.Kopke (2011).Reduced tillage effects on soil properties and growth of cereals and associated weeds under organic farming . Soil and Tillage Research,111:133 - 141.

Verlinden ，G., B.Pycke , J.Mertens ，F. Debersa ，K.Verheyen ， G.Baert , J.Brtes and G. Haesaert (2009). Application of humic substance results in consistent uptake . J.Plant Nutu . 32: 1407-1426 .

Ulukan , H.(2007). Effect of soil applied humic acid at different sowing times on some yield components in wheat (Triticum Spp.) International Journal of Botany 4(2) : 164-175 .

Fayoum J. Agric. Res. \& Dev., Vol. 34, No.2 July, 2020 
Gihan . A. Mohamed et al.,

Wang, X .J.,Z.Q.Wang and S .G.Li (1995). The effect of humic acids on the availability of phosphorus fertilizers in alkaline soils. Soil Use Manage J.,11: 99-102.

Weels, M., S.Reberg - Horton ,A.Smith and J.Grossman (2013). The reduction of plant available nitrogen by cover crop mulches and subsequent effects on soybean performance and weed interference . Agronomy Journal,105 :539-545.

Willian, J.Sarrenson (1997).Financial and economic implication of No-tillage and crop rotation compared to convention cropping system .Mag /GTZ

Zandonadi , D.B., L.P. Canellas and A.R. Facanha (2007) . Indolacetic and humic acids induce root development through a concealed plasma lemma and tonoplast $\mathrm{H}^{+}$pumps activation . Planta . 225: 1583-1595 .

Zhang , W.Z., X.Q. Chen , J.M. Zhou , D.H. Liu,H.Y. Wang and C. W. Du (2013). Influence of humic acid on interaction of ammonium and potassium ions on clay minerals . Pedosphere 23 (4) : 493 -502.

كفاءة أستخدام حمض الهيوميك على أنتاجية محصول القمح وبعض خواص التربة تحت نظام تقليل الخدمة ونقص مياة الرى .

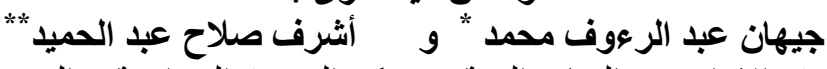

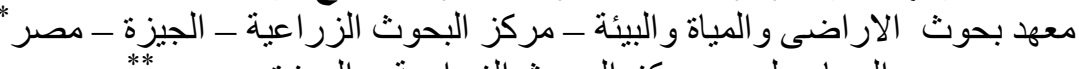

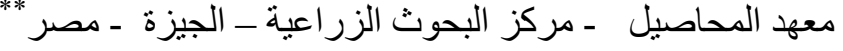

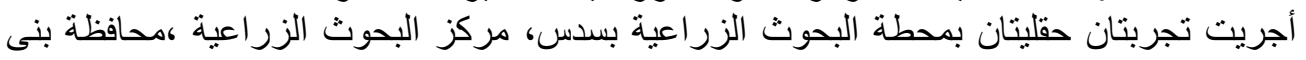

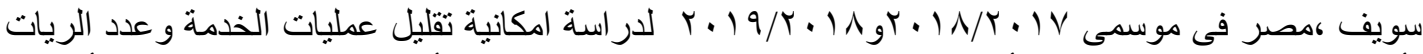

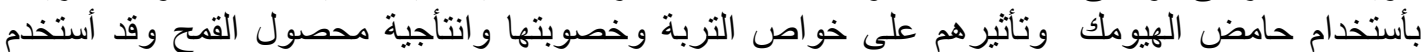

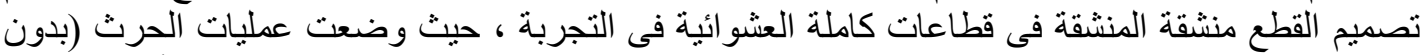

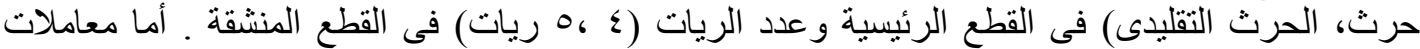

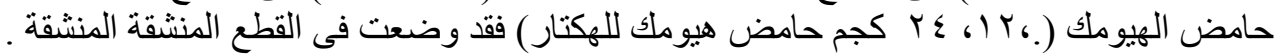

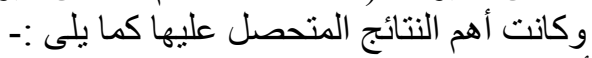

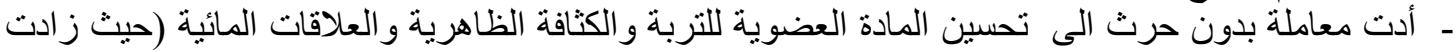

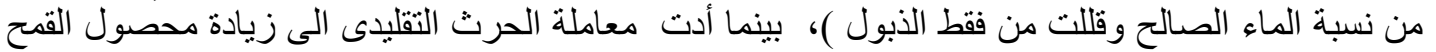

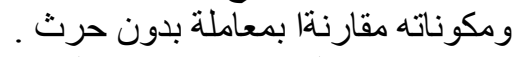

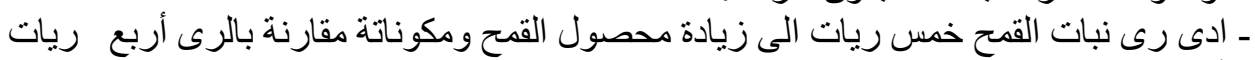

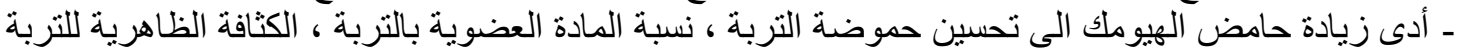
و العلاقات المائية وصلاحية عناصر النيتروجين و الفسفور و البوتاسيوم فى التربة بعد حصاد القية الترح وكذلك الكية

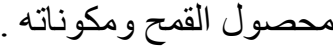

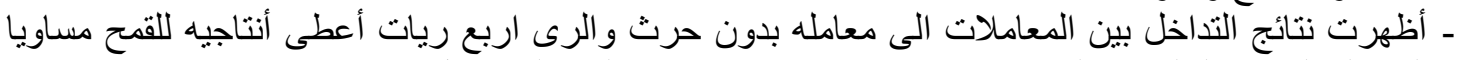

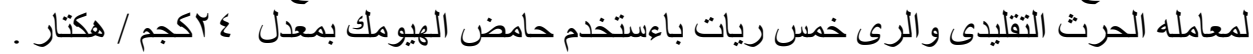

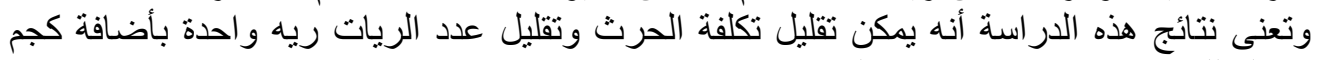

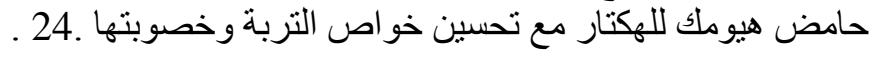

Fayoum J. Agric. Res. \& Dev., Vol. 34, No.2 July, 2020 